\title{
PCOS: a Chronic Disease That Fails to Produce Adequately Specialized Pro-resolving Lipid Mediators (SPMs).
}

Pedro-Antonio Regidor ( $\nabla$ pedro-antonio.regidor@exeltis.com )

Exeltis Healthcare https://orcid.org/0000-0002-9551-2847

\section{Xavier de la Rosa}

Brigham and Women's Hospital

Anna Mueller

Exeltis Healthcare

\section{Manuela Sailer}

Exeltis Healthcare

\section{Fernando Gonzalez Santos}

Solutex

\section{Rafael Gracia Banzo}

Solutex GC

Jose Miguel Rizo

OTC Chemo

\section{Research Article}

Keywords: PCOS, obesity, inflammation, specialized pro-resolving mediators (SPMs)

Posted Date: January 6th, 2022

DOI: https://doi.org/10.21203/rs.3.rs-1214060/v1

License: (c) (i) This work is licensed under a Creative Commons Attribution 4.0 International License. Read Full License

Version of Record: A version of this preprint was published at Biomedicines on February 16th, 2022. See the published version at https://doi.org/10.3390/biomedicines10020456. 


\section{Abstract}

Introduction: Polycystic Ovary Syndrome (PCOS) is an endocrinologic disorder that affects $5-15 \%$ of women of their reproductive age and is a frequent cause of infertility. Major symptoms include hyperandrogenism, ovulatory dysfunction, and often obesity and/or insulin resistance. PCOS also represents a state of chronic low-grade inflammation that is closely interlinked with the metabolic features. "Classical" pro-inflammatory lipid mediators like prostaglandins (PG), leukotrienes (LT), or thromboxanes (TX) are derived from arachidonic acid (AA) and are crucial for the initial response.

Resolution processes are driven by four families of so-called specialized pro-resolving mediators (SPMs): resolvins, maresins, lipoxins, and protectins. The study aimed to establish lipid mediator profiles of PCOS patients compared to healthy women to identify differences in their resolutive and pro-inflammatory lipid parameters.

Material and Methods: Fifteen female patients (18-45 years) were diagnosed with PCOS according to Rotterdam criteria, and five healthy women, as comparator group, were recruited for the study. The main outcome measures were: Pro-inflammatory lipid mediators (PG, LT, TX) and their precursor AA; SPMs (Resolvins, Maresins, Protectins, Lipoxins), their precursors EPA, DHA, DPA, and their active biosynthesis pathway intermediates (18-HEPE, 17-HDHA, 14-HDHA).

Results: The level of pro-inflammatory parameters in serum was significantly higher in PCOS-affected women. The ratio [(sum of pro-inflammatory molecules) / (sum of SPMs plus hydroxylated intermediates)] reflecting the inflammatory state was significantly lower in the group of healthy women.

Conclusion: There is a strong pro-inflammatory state in PCOS patients. Further research will clarify whether supplementation with SPMs or their precursors may improve this state.

\section{Plain English Summary}

Context: Polycystic Ovary Syndrome (PCOS) is an endocrinologic disorder that affects 5-15\% of women in their reproductive age and is a frequent cause for infertility. PCOS also represents a state of chronic low-grade inflammation that is closely interlinked with the metabolic features.

Pro-inflammatory lipid mediators like prostaglandins (PG), leukotrienes (LT) or thromboxanes (TX) are derived from arachidonic acid $(A A)$ and are crucial for the inflammatory response. SPM biosynthesis starts from the essential poly-unsaturated fatty acids DHA, DPA or EPA via certain hydroxylated intermediates (18-HEPE, 17-HDHA, 14-HDHA).

Several chronic diseases have been attributed to insufficient resolution of inflammation and the benefits of SPM supplementation is documented

Objective: To establish lipid mediator profiles of PCOS patients compared to healthy women to identify differences in their resolutive and pro-inflammatory lipid parameters. 
Methods: Fourteen patients (18-45 years) with a diagnosed PCOS according to Rotterdam criteria and 5 healthy women were recruited in one study centre in Germany.

Main Outcome Measure: Pro-inflammatory lipid mediators (PG, LT, TX) and their precursor AA; SPMs (Resolvins, Maresins, Protectins, Lipoxins), their precursors EPA, DHA, DPA and the metabolic intermediates (18-HEPE, 17-HDHA, 14-HDHA).

Results: The level of pro-inflammatory parameters in serum was significantly higher in PCOS-affected women. The ratio [(sum of pro-inflammatory molecules) / (sum of SPMs plus hydroxylated intermediates)] reflecting the inflammatory state was significantly lower in the group of healthy women.

Conclusion: There is a strong pro-inflammatory state in PCOS patients. Further research will clarify whether a supplementation with SPMs or their precursors may improve this state.

\section{Key Clinical Message}

SPMs, which derive from 18-HEPE, 17-HDHA, and 14-HDHA, and are biosynthesized from their respective precursor omega-3-fatty acids EPA and DHA, have a possible influence on the resolution of inflammation associated with polycystic ovary syndrome.

\section{Introduction}

Polycystic Ovary Syndrome (PCOS) is a disease that causes irregular bleeding, chronic anovulation, androgen excess, and a typical ovarian ultrasound feature [1]. It affects between 5 and $10 \%$ of women in their reproductive age, thus representing one of the most frequent causes of infertility [2]. The reasons for the development of PCOS have not been resolved yet. Genetic predisposition, together with the gestational environment and lifestyle factors, seem to be critical contributors [3]. Apart from the cardinal diagnostic criteria including hyperandrogenism, ovulatory dysfunction, and/or the morphology of polycystic ovaries as defined by the so-called "Rotterdam-Criteria" [4], other characteristics are related to the disease. PCOS is often accompanied by obesity [5], and $30-40 \%$ of women with PCOS show a reduced glucose tolerance, often accompanied by insulin resistance [6]. $80 \%$ of obese women and $30-40 \%$ of lean individuals with PCOS suffer from hyperinsulinemia $[6,7]$. It has been found that hyperinsulinemia is a crucial factor in the clinical pathogenesis of PCOS and seems to be independent of weight [8]. Excess insulin may lead to enhanced androgen synthesis by direct stimulation of the androgen production on the one hand and by reducing the serum levels of sex hormone-binding globulin (SHBG) on the other side, thereby contributing to the androgen excess characteristic for PCOS [8]. In addition, obesity has a substantial impact on the severity of PCOS symptoms [9]. Apart from reinforcing insulin resistance, adipocytes show an altered hormone metabolism that contributes to the endocrinologic disorder [10].

Furthermore, excess adipose tissue is a source of chronic low-grade inflammatory processes, and PCOS is considered an inflammatory disease [11]. Inflammatory response has been defined as an ensemble of initiation and active resolution processes. Within this perception, the resolution of inflammation is 
dependent on a class of lipid mediator molecules called specialized pro-resolving mediators (SPM) [12]. These molecules are derived from polyunsaturated fatty acids EPA (Eicosapentaenoic Acid) and DHA (Docosahexaenoic Acid) and are synthesized via specific intermediate molecules by cells of the immune system. This publication will focus on the role of SPMs in chronic inflammatory diseases like PCOS and the potential benefit of supplementation with their precursor molecules.

\section{Obesity, insulin resistance, and inflammation}

A state of chronic systemic inflammation is characteristic of obesity. It can be determined by measuring increased serum levels of inflammatory cytokines and altered frequencies and functions of peripheral blood lymphocytes $[13,14,15]$. These changes are manifested at the tissue level of the adipose-, liver- and other tissue beds $[14,15]$. They might be responsible for comorbidities often related to obesity, such as atherosclerosis, diabetes, and steatohepatitis $[16,17,18,19,20]$. This kind of inflammation is often attributed to irregularities in innate immunity. However, innate and adaptive immune systems are closely interlinked, and consequently, obesity-related inflammation is associated with both processes [21]. For example, in obese individuals, systemic levels of free fatty acids are elevated [10]. These molecules are primary ligands of Toll-like receptors, which are critical regulators of the innate immune response [22, 23]. In this way, the systems, which regulate obesity and inflammation, are linked directly.

A further relationship between inflammation and the metabolic system is visible on the cellular level since adipocytes and macrophages are closely related. Their evolution might be traced back to a conventional primordial precursor cell [24].

It has also been demonstrated that insulin resistance and inflammatory processes are closely linked and may stimulate each other [25]. Both subclinical inflammation and insulin resistance are important markers for the development of cardiovascular disease [26]. For women with PCOS, whose cardiovascular risks are elevated, a connection between inflammation and their hormonal- metabolic features was shown [27].

Since obesity, insulin resistance, and inflammation, key features of PCOS, are correlated, it is worthwhile looking at the possible pathways of inflammation that accompany PCOS, considering the modern perception of inflammatory processes.

The present study was therefore conceived to describe the physiological status of the innate immune response and its resolution potential by blood profiling of eicosanoid parameters and pro-resolving mediators in the plasma and sera of patients suffering from a PCOS as this information is lacking to date and comparing the results with a healthy group to describe the intensity of the pro-inflammatory lipid mediator's exacerbation in the peripheral blood in these patients.

\section{Material And Methods}

\section{Human plasma and serum of PCOS patients}


Fifteen PCOS patients were evaluated in this study. A control group of five healthy patients was used for comparison. The healthy patients were probands with no evident clinical acute disease or known pathological anamnesis in the medical history. The BMI of both groups was similar.

All patients were recruited at the Lubos Klinik in Munich, Germany.

Samples were obtained at 8 am under fasting conditions.

Table 1 shows the demographic data of the patients.

The whole study was performed following a protocol designed and conducted following the ethical principles that have their origin in the Declaration of Helsinki and are consistent with GCP and existing regulatory requirements. Institutional review board approval was obtained from the study site.

\section{Ethical approval}

Ethical approval was obtained for the investigational centre. The overall approval for the study was given on Jul 17, 2020, by the Ethical Committee of the Bayerischen Landesärztekammer; Nr. 20056.

Clinical trial registration: DRKS-ID: DRKS00022337. Date of registration: Jun 29, 2020.

\section{Blood sample analyses.}

Blood samples of plasma and serum were obtained for each patient. Each of these samples was considered a mono-replicate.

After standard preliminary treatment, samples were stored at -80 degrees Celsius until they were processed in the laboratory. They were all analysed separately.

\section{Lipid mediator extraction and profiling (LC-MS/MS)}

Lipid mediators were extracted from human plasma and serum samples following the SPE (Solid Phase Extraction) method described below. Internal labelled standards $d_{8}-5-H E T E, d_{5}-R^{2} D 2, d_{5}-L_{X A}, d_{4}-L_{T} B_{4}, d_{4}-$ $\mathrm{PGE}_{2}$ (500 pg each, Cayman Chemical Company) in $4 \mathrm{~mL}$ of methanol (Methanol Optima LC/MS Grade, Fisher Chemical) were added to each sample (plasma or serum, $1 \mathrm{~mL}$ ) previously thawed on ice. These labelled standards were used for the amount determined and the calculations of the recovery of the lipid mediators. Next, the samples were placed at $-80^{\circ} \mathrm{C}$ for 30 minutes to allow the precipitation of proteins. The probes were centrifugated in the following working step $\left(2000 \mathrm{~g}, 10 \mathrm{~min}, 4^{\circ} \mathrm{C}\right)$. The supernatants were obtained from each sample, and SPE was carried out according to optimized and reported methods [28, 29]. Samples were rapidly acidified to $\mathrm{pH}=3.5$ with $9 \mathrm{~mL}$ of acidic water $(\mathrm{HCl})$ before loading onto SPE columns (100mg, $10 \mathrm{~mL}$, Biotage) and pH neutralized with $4 \mathrm{~mL}$ of MiliQ water, followed by $4 \mathrm{~mL}$ of $\mathrm{n}$ hexane wash step. After, compounds were eluted with $9 \mathrm{~mL}$ of methyl format. Extracts from the SPE were brought to dryness under a gentle stream of nitrogen and immediately resuspended in methanol/water 
(50:50 vol/vol) (MeOH/Water Optima LC/MS Grade, Fisher Chemical, both) before injection into an LCMS/MS system.

\section{Targeted LC-MS/MS Acquisition Parameters}

LC-MS/MS system consisting of a Qtrap 5500 (Sciex) equipped with a Shimadzu LC-20AD HPLC pump. A Kinetex Core-Shell LC-18 column (100 mm $\times 4.6 \mathrm{~mm} \times 2.6 \mu \mathrm{m}$, Phenomenex) was kept in a column oven maintained at $50{ }^{\circ} \mathrm{C}$. A binary eluent system of LC-MS/MS grade water (A) (Fisher Chemical) and LCMS/MS grade methanol (Fisher Chemical) (B), both with $0.01 \%(\mathrm{v} / \mathrm{v})$ of acetic acid, was used as mobile phase. LMs were eluted in a gradient program respect to the composition of $B$ is as follows: $0-2$ min, $50 \%$; 2-14.5 min, $80 \%$; $14.6-25 \mathrm{~min} ; 98 \%$. The flow rate was $0.5 \mathrm{~mL} / \mathrm{min}$.

The QTRAP 5500 was operated in negative ionization mode, using scheduled Multiple Reaction Monitoring (MRM) coupled with the information-dependent acquisition (IDA) and an Enhanced Product Ion scan (EPI). Each LM parameter (CE, target retention time (RT), and specific Q1 and Q3 mass) was optimized according to reported methods $[29,30]$. To monitor and quantify LMs of interest, quantities were taken as areas under the peak. We used MRM with MS/MS matching signature ion fragments for each molecule (at least six diagnostic ions; $<0.1$ picograms was considered below the limit of detection) using published criteria [30]. Examples of representative MRM spectra are presented in figure 5. The laboratory analyses were performed at Solutex GC SL.

\section{Statistical analyses}

Quantitative measurements were presented as mean, standard error, minimum and maximum. When indicated, Outlier exclusion was calculated using default parameters ROUT $(\mathrm{Q}=1 \%)$ from GraphPad Prism version 9.0.2, GraphPad Software, San Diego, California USA. Comparisons were consequently made using an unpaired one-tailed $t$-test.

All tests were done with a one-tailed t-test, and statistical significance was considered at $P<0.05$. We did not make any adjustments for multiple testing; thus, the results are explorative and descriptive.

A ratio between pro-inflammatory and pro resolutive parameters was established to describe the physiology of both investigated axes (pro-inflammatory and pro resolutive).

The proposed ratios have the purpose of seeking the overall balance/unbalance of interconnected metabolic routes and the overall status of the resolution of the immune response.

\section{Evaluated parameters}

Fatty acids (EPA, DHA, ARA, DPA); SPM monohydroxylated-containing precursors (17-HDHA, 18-HEPE, 14HDHA); SPM's (Resolvins: RvE1, RvD1, RvD2, RvD3, RvD4, RvD5; Maresins: MaR1, MaR2; Protectins: PD1, PDX; Lipoxins: $\left.\mathrm{LXA}_{4}, \mathrm{LXB}_{4}\right)$. Eicosanoids: Prostaglandins ( $\left.\mathrm{PGE}_{2}, \mathrm{PGD}_{2}, \mathrm{PGF}_{2 \mathrm{a}}\right)$ Thromboxanes $\left(\mathrm{TxB}_{2}\right)$

Leukotrienes $\left(\mathrm{LTB}_{4}\right)$ 


\section{Results}

In this observational study, we observe that the quantity of each parameter was detectable in the sera but not in the same way in the participants' plasma.

After quantitation, summation of total ARA-derived pro-inflammatory mediators resulted in a statistically significant increase $(P<0.05)$ when comparing sera from PCOS- patients with healthy subjects. These proinflammatory mediators include LTB4, PGD2, PGE2, PGF2, and TXB2, and values altogether were 100 times higher compared to those of healthy subjects (see figure 1). Measured prostanoids, including PGD2, PGE2, and PGF2, all together were increased by $600 \%$ in serum from patients with PCOS compared to healthy subjects (figure 1$)$. Thromboxane TXB2 was also statistically significantly $(P<0.05)$ higher in the serum from patients diagnosed with PCOS as compared to healthy subjects, which may reflect that these patients could suffer from coagulopathies (figure 1).

We next studied whether these patients might have a disbalance in SPM formation. Specifically, comparing the ratio of total pro-inflammatory lipid mediators, including LTB4, PGD2, PGE2, PGF2, and TXB2 vs. total SPMs formed were statistically significantly different between the test groups (figure 1). We observed in serum that the ratio of complete pro-inflammatory lipid mediators to the summation of SPMs, including 14-HDHA, 17-HDHA, and 18-HEPE, was statistically higher for patients with a PCOS than those observed in the serum of healthy subjects $(P<0.05)$. This finding suggested that infections could impair resolution mechanism(s) due to exacerbated inflammation.

We quantified the free-fatty-acid-precursors of resolving mediators. We observed that DHA, DPA, EPA, and ARA were not statistically significantly higher in PCOS patients' plasma and serum (figure 2) as compared to those of healthy subjects. Interestingly, we observed that PCOS patients presented statistically more elevated amounts of the ratio pro-inflammatory parameters / SPMs, including the monohydroxylates in the serum compared to the plasma. (figure3).

As shown in figure 1, the mean value of total prostanoids in the serum was $30.000 \mathrm{pg} / \mathrm{ml}$ in healthy subjects and $60000 \mathrm{pg} / \mathrm{ml}$ in PCOS patients (see figure 1). When comparing the differences for the thromboxane values between the healthy subjects and the PCOS patients, statistically significant differences could also be observed. PCOS patients expressed significantly higher values than the healthy controls (see figure 1).

Figures 4 (plasma) and 5 (serum) show in a graphical way the heat maps of the metalipidinomic results.

Human plasma or serum was extracted using SPE and subject to targeted LC-MS/MS (see method above). Targeted LM and pathway markers were profiled. Each mediator was identified using published criteria obtained on their structure, including identification criteria of at least six characteristic diagnostic ions present in their MS-MS spectra. Representative screen captures of MS-MS enhanced product ion (EPI) spectra captured from the chromatographic region of (A) LTB4 (B) PGD2 (C) PGE2 (D) PGF2 (E) 
TXB2 (F) RvD1 (G) PD1 (H) MaR1 and (I) MaR2. Screen captures were taken using SCIEX OS software. Insets: Chemical structures and prominent fragmentations.

Table 2 depicts all the values in a tabular form.

\section{Discussion}

This study described the significant disbalance between pro-inflammatory eicosanoid- derived lipid mediators and pro-resolutive markers in the serum of PCOS patients. PCOS represents a chronic inflammatory condition since classical indicators for an inflammatory response are present, such as increased values of IL-6, C-reactive protein, fibrinogen, and erythrocyte sedimentation rate $(11,31)$. The presented data supports this concept, as the lipidome of PCOS patients is shifted towards the proinflammatory axis with an increase in pro-inflammatory prostanoid derivatives and an elevated ratio of [pro-inflammatory LM]/ [the sum of SPMs and their hydroxylated precursors].

Some widespread diseases like diabetes, cardiovascular disease, and obesity are associated with chronic inflammation $[15,17,32]$. These pathologies are strongly interlinked with diet, and the positive impact of polyunsaturated fatty acids (PUFA) - rich diet on cardiovascular health is broadly accepted [33]. Both EPA and DHA show an anti-inflammatory effect, and in this context, their role as precursors for SPM biosynthesis has been discussed $[34,35]$. The crucial role of SPMs in such chronic inflammatory states has become evident throughout the past years, and the underlying molecular mechanisms are increasingly elucidated $[12,39]$

For the DHA-derived SPM protectin PD 1 and its hydroxylated precursors, for example, a positive influence on the metabolism of fatty tissue was demonstrated, suggesting a potential role in the management of obesity [36]. For DHA-derived SPM RvD1, a molecular mechanism for its possible cardioprotective effect has been demonstrated: it can activate lipoxin A4/formyl peptide receptor 2 (ALX/FPR2), which serves as a sensor for the resolution of inflammation in the context of coronary heart disease. In animal experiments, ALX7FPR-null mice developed obesity, diastolic dysfunction and showed reduced SPMlevels associated with an impaired resolution of inflammation after cardiac injury [37].

SPM biosynthesis is, off-course, based on the abundance of their PUFA-precursors. However, in several experimental setups, the SPM biosynthesis was disturbed by altered activities of the involved enzymes [36]. Depending on the affected enzyme, supplementation with the hydroxylated intermediates of the SPM biosynthetic pathways may be efficacious in those cases, as demonstrated in a setting with leukocytes of obese individuals that showed a profound deficiency in the biosynthesis of RvD: incubating the leukocytes with 17-HDHA, the precursor of RvD, restored the SPM production [38].

Therefore, supplementation with DHA- or EPA-derived SPMs and their corresponding hydroxylated precursor metabolites 18-HEPA, 17-HDHA, and 14-HDHA may represent a promising approach to address the pathologic features often associated with PCOS: hyperandrogenemia, obesity, and insulin resistance all aggravate each other and are all associated with chronic inflammatory processes. In the present study, 
an association with the lipid mediator profile was demonstrated that was significantly shifted towards the pro-inflammatory axis compared to healthy women. Exciting was the greatly increased level of thromboxane TXB2 in PCOS patients compared to the test group, as its precursor, TXA2, plays an essential role in platelet activation and aggregation. PCOS-affected women are known to have a 2-fold increased risk for venous thromboembolic events compared to healthy women.

Our study supports the former investigation of Duleva et al. [40] and Rudnika et al. [41] that could demonstrate using classical inflammatory markers that PCOS is an inflammatory disease.

The fact that white blood cells and cytokines, and interleukins are elevated could be reaffirmed with our data showing a metalipidinomic disbalance in women with PCOS.

Treatment of PCOS is mainly focused on weight loss, use of anti-androgenic hormone preparations, treatment with insulin-sensitizing agents or the whole repertoire of ovulation induction, and in vitro fertilization techniques when child wish comes into focus. At the same time, the underlying inflammatory processes are relatively neglected as a therapeutic target [42].

\section{Conclusion}

As the SPMs, which derive from 18-HEPE, 17-HDHA, and 14-HDHA, and are biosynthesized from their respective precursor omega-3-fatty acids EPA and DHA, have a possible influence on the resolution of inflammation associated with polycystic ovary syndrome a targeted supplementation with SPMs or their precursors may be a valuable novel therapeutic strategy worth further investigation in the management of women with a PCOS.

Especially as this study could show the lack of SPMs in the women's combined with an overexpression po pro inflammatory substance's and also thromboxanes This finding could also explain the 2-fold higher risk of thromboembolic events that is associated with the PCOS syndrome.

\section{Abbreviations}

PCOS = Polycystic Ovary Syndrome

SPM = Specialized Pro Resolving Lipid Mediators

$P G=$ Prostaglandines

$\mathrm{LT}=$ Leukotrienes

$\mathrm{TX}=$ Thromboxanes

$\mathrm{AA}=$ Arachidonic Acid

$\mathrm{EPA}=$ Eicosapentaenoic Acid 
DHA = Docosahexaenoic Acid

DPA = Docosapentanoic Acid

18-HEPE = 18-Hydroxy Eicosapentaenoic

17-HDHA = 17-Hydroxy Docosahexaenoic Acid

14-HDHA = 17-Hydroxy Docosahexaenoic Acid

$\mathrm{BMI}=$ Body Mass Index

GCP $=$ Good Clinical Practice

LC-MS/MS = Lipid mediator extraction and profiling

SPE $=$ Solid Phase Extraction

$\mathrm{HCl}=$ Acid water

MRM $=$ Multiple Reaction Monitoring

IDA = Information-dependent acquisition

EPI $=$ Enhanced Product Ion

LM = Lipid Mediator

\section{Declarations}

Funding: Insud Pharma

Conflict of Interest: Pedro-Antonio Regidor is an employee of Exeltis Healthcare, Anna Müller and Manuela Sailer are employees of Exeltis Germany, Fernando Gonzalez Santos and Rafael Gracia Banzo are employees of Solutex Spain, Jose Miguel Rizo is an employee of Chemo OTC Spain. Xavier de la Rosa declares no conflict of interest

Ethical approval: Ethical approval was obtained from the investigational center. The overall approval for the study was given on Jul 17,2020, by the Ethical Committee of the Bayerischen Landesärztekammer; Nr. 20056.

Data availability: Not applicable.

Author Contribution

Pedro-Antonio Regidor: Responsible for the concept of resolution of inflammation in PCOS 
Anna Müller: Responsible for literature research and writing of the manuscript

Manuela Sailer: Responsible for graphic design

Fernando Gonzalez Santos: Responsible for the development of SPMs use in humans and therefore for essential scientific data

Jose Miguel Rizo: responsible for data acquisition

Rafael Gracia Banzo: Responsible for laboratory analyses

Xavier de la Rosa: Responsible for coordination of the manuscript

Consent for publication: The participants allowed and gave their consent for publication of the obtained laboratory data including the clinical characteristics according to the data protection law of the European Union and Germany.

Acknowledgement: We thank Julio Boza for correcting the manuscript and creating the graphs.

\section{References}

1. Ehrmann DA. Polycystic ovary syndrome. NEJM. 2005; 352 (12): 1223 - 1236.

2. Homburg R. Polycystic ovary syndrome-from gynecological curiosity to multisystem endocrinopathy. Human Reproduction. 1996; 11(1): 29-39.

3. Diamanti-Kandarakis E, Kandarakis H, Legro RS. The role of genes and environment in the etiology of PCOS. Endocrine. 2006; 30 (1): 19-26.

4. Rotterdam ESHRE/ASRM-Sponsored PCOS Consensus Workshop Group, Revised 2003 consensus on diagnostic criteria and long-term health risks related to polycystic ovary syndrome (PCOS). Human Reproduction. 2004; 19 (1): 41-47.

5. Sam S. Obesity and Polycystic Ovary Syndrome. Obes Manag. 2007;3(2): 69-73.

6. Ehrmann DA, Barnes RB, Rosenfield RL, Cavaghan MK, Imperial J. Prevalence of impaired glucose tolerance and diabetes in women with polycystic ovary syndrome. Diabetes Care. 1999; 22(1): 141146.

7. Ciampelli M, Fulghesu AM, Cucinelli F, Pavone V, Ronsisvalle E, Guido M, Caruso A, Lanzone A. Impact of insulin and body mass index on metabolic and endocrine variables in polycystic ovary syndrome. Metabolism: Clinical and Experimental. 1999; 48(2): 167-172.

8. Diamanti-Kandarakis E, Dunaif A. Insulin resistance and the polycystic ovary syndrome revisited: an update on mechanisms and implications. Endocr Rev. 2012; 33(6): 981-1030.

9. Teede H, Deeks A, Moran L. Polycystic ovary syndrome: a complex condition with psychological, reproductive, and metabolic manifestations that impacts on health across the lifespan. BMC Med. 2010; 8 (1): 41. 
10. Rojas J, Chávez M, Olivar L, Rojas M, Morillo J, Mejías J, Calvo M, Bermúdez V. Polycystic ovary syndrome, insulin resistance, and obesity: navigating the pathophysiologic labyrinth. Int J Reprod Med.

11. Rudnicka E, Kunicki M, Suchta K, Machura P, Grymowicz M, Smolarczyk R. Inflammatory Markers in Women with Polycystic Ovary Syndrome. Biomed Res Int. 2020 Mar 4; 2020:4092470.

12. Serhan CN. Pro-resolving lipid mediators are leads for resolution physiology. Nature 2014; 510: $92-$ 101.

13. Bastard JP, Jardel C, Bruckert E, Blondy P, Capeau J, Laville M, Vidal H, Hainque B. Elevated levels of interleukin 6 are reduced in serum and subcutaneous adipose tissue of obese women after weight loss. Journal of Clinical Endocrinology and Metabolism. 2000; 85 (9): 3338-3342.

14. O’Rourke RW, Kay T, Lyle EA, Traxler SA, Deveney CW, Jobe BA, Roberts CT Jr, Marks D, Rosenbaum JT. Alterations in peripheral blood lymphocyte cytokine expression in obesity. Clinical and Experimental Immunology. 2006; 146 (1): 39- 46.

15. Festa A, D’Agostino R Jr., Williams K, Karter AJ, Mayer-Davis EJ, Tracy RP, Haffner SM. The relation of body fat mass and distribution to markers of chronic inflammation. International Journal of Obesity. 2001; 25 (10): 1407-1415.

16. DiehI MAEA. Nonalcoholic steatosis and steatohepatitis IV. Nonalcoholic fatty liver disease abnormalities in macrophage function and cytokines. American Journal of Physiology. 2002; 282 (1): $1-5$.

17. Arkan MC, Hevener AL, Greten FR, Maeda S, Li Z-W, Long JM, Wynshaw-Boris A, Poli G, Olefsky J, Karin M. IKK- $\beta$ links inflammation to obesity-induced insulin resistance. Nature Medicine. 2005; 11(2): 191-198.

18. Schönbeck U, Mach F, Sukhova GK, Herman M, Graber P, Kehry MR, Libby P. CD40 ligation induces tissue factor expression in human vascular smooth muscle cells. American Journal of Pathology. 2000; 156(1): 7-14.

19. Reardon CA, Getz GS. Mouse models of atherosclerosis. Current Opinion in Lipidology. $2001 ; 12$ (2): 167-173.

20. Michelsen KS, Wong MH, Shah PK, Zhang W, Yano J, Doherty TM, Akira S, Rajavashisth TB, Arditi M. Lack of Toll-like receptor 4 or myeloid differentiation factor 88 reduces atherosclerosis and alters plaque phenotype in mice deficient in apolipoprotein E. Proceedings of the National Academy of Sciences of the United States of America. 2004; 101 (29): 10679-10684.

21. Cancello R, Tordjman J, Poitou C, Guilhem G, Bouillot JL, Hugol D, Coussieu C, Basdevant A, Bar Hen A, Bedossa P, Guerre-Millo M, Clément K. Increased infiltration of macrophages in omental adipose tissue is associated with marked hepatic lesions in morbid human obesity. Diabetes. 2006; 55 (6): 1554-1561.

22. Davis JE, Gabler NK, Walker-Daniels J, Spurlock ME. TIr-4 deficiency selectively protects against obesity induced by diets high in saturated fat. Obesity. 2008; 16 (6): 1248-1255. 
23. Schaeffler A, Gross P, Buettner R, Bollheimer C, Buechler C, Neumeier M, Kopp A, Schoelmerich J, Falk W. Fatty acid-induced induction of Toll-like receptor- 4 /nuclear factor-KB pathway in adipocytes links nutritional signalling with innate immunity. Immunology. 2009; 126 (2): 233-245.

24. Charrière G, Cousin B, Arnaud E, André M, Bacou F, Penicaud L, Casteilla L. Preadipocyte conversion to macrophage: evidence of plasticity. Journal of Biological Chemistry. 2003; 278 (11): 9850-9855.

25. Bloomgarden ZT. Inflammation and insulin resistance. Diabetes Care 2003; 26 (6): 1922-1926.

26. Frishman WH. Biologic markers as predictors of cardiovascular disease. American Journal of Medicine. 1998; 104 (6A): 18S-27S.

27. Orio Jr. F, Palomba S, Cascella T, Sebastiano Di Biase S, Manguso F, Tauchmanovà L, Nardo LG, Labella D, Savastano S, Russo T, Zullo F, Colao A, Lombardi G. The increase of leukocytes as a new putative marker of low-grade chronic inflammation and early cardiovascular risk in polycystic ovary syndrome. Journal of Clinical Endocrinology and Metabolism. 2005; 90 (1): 2-5.

28. Flower R J. Prostaglandins, bioassay, and inflammation. Br. J. Pharmacol. 2006 Jan; 147 Suppl.1: S82-192.

29. Chiurchiù V, Leuti A, Maccarrone M. Bioactive Lipids and Chronic Inflammation: Managing the Fire Within. Front Immunol. 2018 Jan 29; 9:38.

30. Dinarello CA. Anti-inflammatory Agents: Present and Future. Cell. 2010 Mar 19;140(6):935-50.

31. Patel, S. Polycystic ovary syndrome (PCOS), an inflammatory, systemic, lifestyle endocrinopathy. J. Steroid Biochem. Mol. Biol. 2018, 182, 27-36.

32. Wellen KE, Hotamisligil GS. Inflammation, stress, and diabetes. J Clin Invest. 2005 May; 115(5):11111119.

33. Schwab U, Lauritzen L, Tholstrup T, et al. effect of the amount and type of dietary fat on cardiometabolic risk factors and risk of developing type 2 diabetes, cardiovascular diseases, and cancer: a systematic review. Food Nutr Res. 2014; 58:10.3402/fnr. v58.25145. 10. doi:10.3402/fnr. v58.25145

34. Calder, P.C. Omega-3 polyunsaturated fatty acids and inflammatory processes: nutrition or pharmacology? Br. J. Clin. Pharmacol. 2013, 75, 645-662.

35. Regidor PA, Mueller A, Sailer M et al. Chronic Inflammation in PCOS: The Potential Benefits of Specialized Pro-Resolving Lipid Mediators (SPMs) in the Improvement of the Resolutive Response. Int J Mol Sci. 2020;22 (1):384. DOl: 10.3390/ijms22010384.

36. Hansen TV, Vik A, Serhan CN. The protectin family of specialized pro-resolving mediators. Potent immunoresolvents enabling innovative approaches to target obesity and diabetes. Front Pharmacol. 2019 Jan 17; 9: 1582.

37. Tourki B, Kain V, Pullen AB, Norris PC, Patel N, Arora P, Leroy X, Serhan CN, Halade GV. Lack of resolution sensor drives age-related cardiometabolic and cardiorenal defects and impedes inflammation-resolution in heart failure. Molecular Metabolism. 2020 Jan; 31:138 - 149.

38. López-Vicario C, Titos E, Walker ME, Alcaraz-Quiles J, Casulleras M, Durán-Güell M, Flores-Costa R, Pérez-Romero N, Forné M, Dalli J, Clària J. Leukocytes from obese individuals exhibit an impaired 
SPM signature. The FASEB Journal. 2019; 33 (6): 7072-7083.

39. López-Vicario, C.; Rius, B.; Alcaraz-Quiles, J.; García-Alonso, V.; Lopategi, A.; Titos, E.; Clària, J. proresolving mediators produced from EPA and DHA: Overview of the pathways involved and their mechanisms in metabolic syndrome and related liver diseases. Eur. J. Pharmacol. 2016, 785, 133143.

40. Duleba AJ, Dokras A. Is PCOS an inflammatory process? Fertil Steril 2012, 97(1): 7-12. DOI: 10.1016/j.fertnstert.2011.11.023.

41. E. Rudnicka E, Kunicki M, Suchta K, Machura P, Grymowicz M, Smolarczyk R. Inflammatory Markers in Women with Polycystic Ovary Syndrome. 2020 Mar 4; 2020:4092470. doi:

10.1155/2020/4092470. eCollection 2020.

42. European Society of Human Reproduction and Embryology. International evidence-based guideline for the assessment and management of polycystic ovary syndrome 2018.

https://www.eshre.eu/Guidelines-and-Legal/Guidelines/Polycystic-Ovary-Syndrome; last access: August 2021.

\section{Tables}

Table 1: Demographical data of the study population.

\begin{tabular}{|lll|}
\hline & Healthy $(\mathrm{n}=5)$ & PCOS $(\mathrm{n}=15)$ \\
\hline Sex & Female $(5)$ & Female $(15)$ \\
\hline Age (years) & $32(29-34)$ & $31(24-42)$ \\
\hline Weight $(\mathrm{kg})$ & $60,7(50-76)$ & $85,8(54-110)$ \\
\hline High $(\mathrm{cm})$ & $167,4(162-173)$ & $164,9(150-175)$ \\
\hline BMI $\left(\mathrm{kg} / \mathrm{m}^{2}\right)$ & $21,5(17.9-28.3)$ & $31,7(19.8-41.9)$ \\
\hline PCOS & NA & $+(15)$ \\
\hline
\end{tabular}

Table 2: ARA, DHA, EPA, and DPA metabolome: healthy and PCOS lipid mediators amount from human plasma and serum. 


\begin{tabular}{|c|c|c|c|c|c|c|c|c|}
\hline & Pcos & PCOS & Healthy & Healthy & PCOS & PCOS & Healthy & Healthy \\
\hline & Plasma & Plasma & Plasma & Plasma & Serum & Serum & Serum & Serum \\
\hline & Averages & SD & Averages & SD & Averages & SD & Averages & SD \\
\hline \multicolumn{9}{|l|}{ Markers } \\
\hline $\begin{array}{l}\text { EPA } \\
(\mathrm{ng} / \mathrm{mL})\end{array}$ & 70,1 & 29,1 & 42,7 & 15,2 & 103,6 & 35,8 & 77,0 & 14,5 \\
\hline $\begin{array}{l}\text { DHA } \\
\text { (ng/mL) }\end{array}$ & 42,2 & 21,3 & 67,5 & 42,9 & 54,6 & 35,4 & 65,1 & 24,4 \\
\hline $\begin{array}{l}\text { DPA } \\
\text { (ng/mL) }\end{array}$ & 89,0 & 41,5 & 57,4 & 33,0 & 113,8 & 44,7 & 85,0 & 29,7 \\
\hline $\begin{array}{l}\text { ARA } \\
(\mathrm{ng} / \mathrm{mL})\end{array}$ & 69,9 & 21,0 & 82,7 & 34,2 & 124,9 & 64,6 & 105,8 & 34,7 \\
\hline $\begin{array}{l}18- \\
\text { HEPE } \\
(\mathrm{ng} / \mathrm{mL})\end{array}$ & 0,4 & 0,2 & 0,2 & 0,1 & 4,0 & 3,6 & 2,4 & 1,3 \\
\hline $\begin{array}{l}\text { 17- } \\
\mathrm{HDHA} \\
(\mathrm{ng} / \mathrm{mL})\end{array}$ & 0,6 & 0,3 & 0,3 & 0,2 & 11,8 & 5,9 & 7,1 & 3,0 \\
\hline $\begin{array}{l}14- \\
\text { HDHA } \\
(\mathrm{ng} / \mathrm{mL})\end{array}$ & 1,6 & 1,2 & 2,0 & 1,3 & 79,8 & 31,8 & 61,2 & 27,4 \\
\hline $\begin{array}{l}\text { RvE1 } \\
\text { (pg/mL) }\end{array}$ & 97,6 & 254,0 & 39,1 & 87,4 & 188,4 & 317,1 & 170,4 & 381,1 \\
\hline $\begin{array}{l}\text { RvD2 } \\
\text { (pg/mL) }\end{array}$ & 1,9 & 2,1 & 3,7 & 8,3 & 4,8 & 13,1 & 0,0 & 0,0 \\
\hline $\begin{array}{l}\text { RvD3 } \\
\text { (pg/mL) }\end{array}$ & 1,1 & 0,9 & 0,7 & 1,7 & 0,7 & 1,4 & 0,9 & 1,4 \\
\hline $\begin{array}{l}\text { RvD1 } \\
\text { (pg/mL) }\end{array}$ & 1,9 & 2,1 & 3,2 & 6,0 & 3,9 & 5,1 & 2,5 & 3,8 \\
\hline $\begin{array}{l}\text { RvD4 } \\
\text { (pg/mL) }\end{array}$ & 0,3 & 0,6 & 0,0 & 0,0 & 1,1 & 2,4 & 0,0 & 0,0 \\
\hline $\begin{array}{l}\text { RvD5 } \\
\text { (pg/mL) }\end{array}$ & 2,7 & 3,2 & 5,7 & 5,9 & 83,7 & 44,4 & 34,2 & 13,1 \\
\hline $\begin{array}{l}\text { Mar-1 } \\
(\mathrm{pg} / \mathrm{mL})\end{array}$ & 38,0 & 36,4 & 7,7 & 11,7 & 81,8 & 77,3 & 21,5 & 27,9 \\
\hline $\begin{array}{l}\text { Mar-2 } \\
(\mathrm{pg} / \mathrm{mL})\end{array}$ & 2,5 & 2,7 & 1,2 & 1,8 & 231,0 & 164,8 & 93,0 & 51,4 \\
\hline $\begin{array}{l}\text { PD1 } \\
\text { (pg/mL) }\end{array}$ & 1,3 & 2,3 & 5,9 & 8,1 & 3,7 & 4,3 & 7,4 & 3,2 \\
\hline
\end{tabular}




\begin{tabular}{|c|c|c|c|c|c|c|c|c|}
\hline $\begin{array}{l}\text { PDX } \\
\text { (pg/mL) }\end{array}$ & 7,5 & 9,2 & 7,4 & 10,4 & 72,1 & 33,1 & 41,6 & 28,7 \\
\hline $\begin{array}{l}\text { LXA4 } \\
(\mathrm{pg} / \mathrm{mL})\end{array}$ & 1,0 & 1,1 & 2,2 & 2,6 & 1,3 & 2,4 & 1,6 & 1,6 \\
\hline $\begin{array}{l}\mathrm{LXB} 4 \\
(\mathrm{pg} / \mathrm{mL})\end{array}$ & 2,0 & 5,8 & 0,0 & 0,0 & 2,0 & 5,6 & 2,7 & 6,0 \\
\hline $\begin{array}{l}\text { PGE2 } \\
\text { (pg/mL) }\end{array}$ & 32,9 & 46,9 & 21,5 & 15,6 & 716,3 & 295,6 & 525,8 & 375,0 \\
\hline $\begin{array}{l}\text { PGD2 } \\
\text { (pg/mL) }\end{array}$ & 5,2 & 4,1 & 6,1 & 4,1 & 58,9 & 18,0 & 51,8 & 20,1 \\
\hline $\begin{array}{l}\text { PGF2a } \\
\text { (pg/mL) }\end{array}$ & 25,6 & 14,6 & 33,1 & 44,7 & 1275,9 & 578,1 & 777,4 & 474,8 \\
\hline $\begin{array}{l}\text { TXB2 } \\
\text { (pg/mL) }\end{array}$ & 511,7 & 592,1 & 275,4 & 147,5 & 54730 & 24475 & 32555 & 16236 \\
\hline $\begin{array}{l}\text { LTB4 } \\
\text { (pg/mL) }\end{array}$ & 51,3 & 90,4 & 28,0 & 14,8 & 273,6 & 160,4 & 211,5 & 74,7 \\
\hline
\end{tabular}

Figures

Figure 1

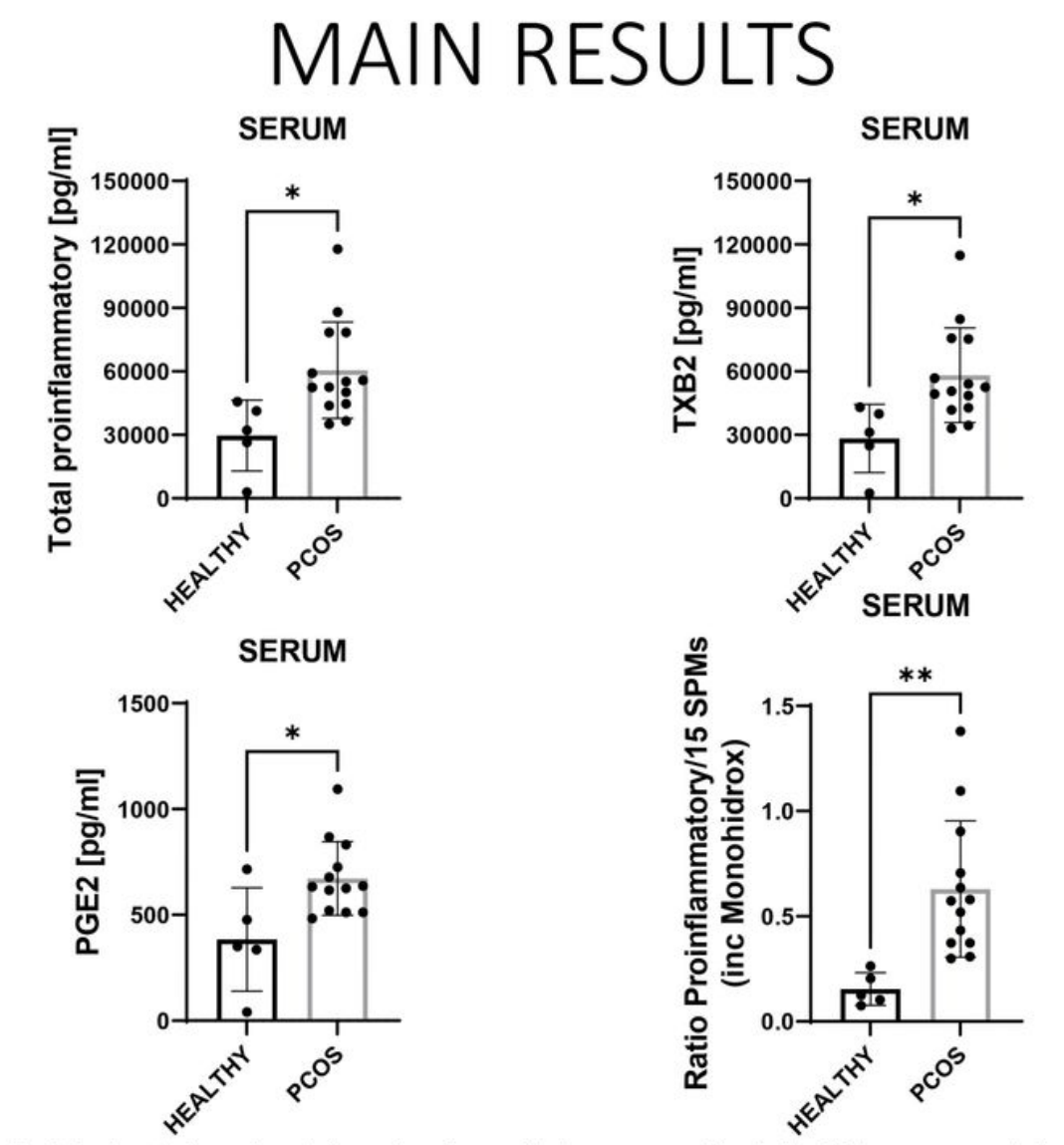

Statistical analysis consisted of a series of two-tailed t-tests assuming similar SD between populations,

Figure 1 
Results of the ARA-derived proinflammatory mediators of the healthy and PCOS patients.

Figure 2

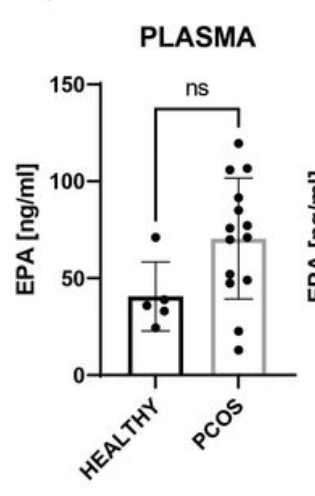

\section{SERUM}

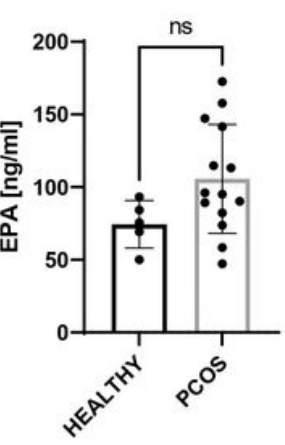

PLASMA

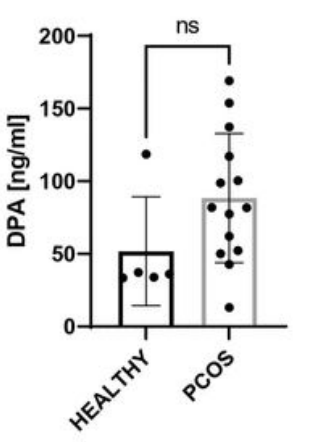

SUBSTRATES
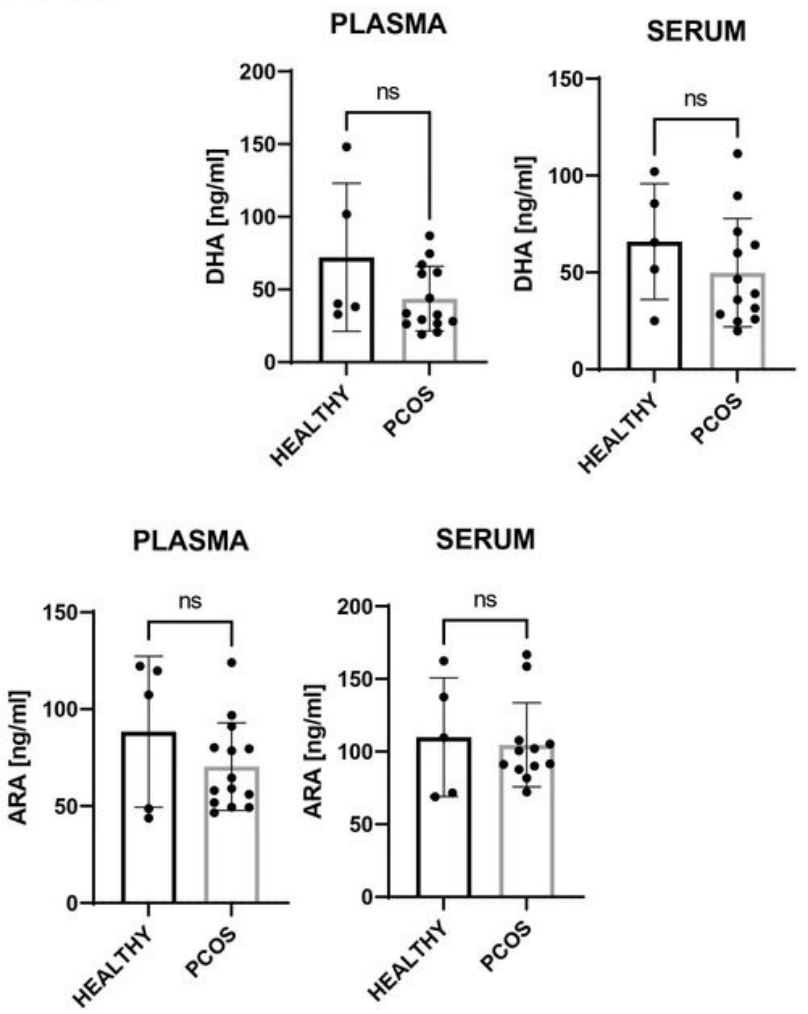

PLASMA

SERUM

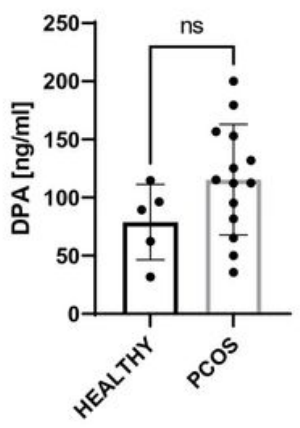

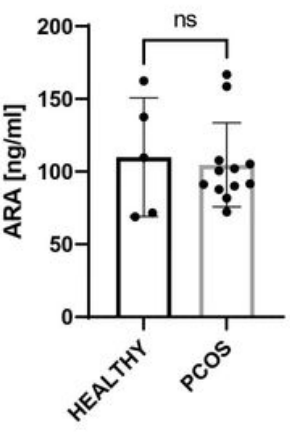

Figure 2

Results of the quantified the free-fatty-acid-precursors of resolving mediators. 
Figure $3 \underset{\text { PLASMA }}{\text { PROINFLAMMMTMATORY VS. RESOLUTION }} \underset{\text { PLASMA }}{\operatorname{R} A T I O S}$
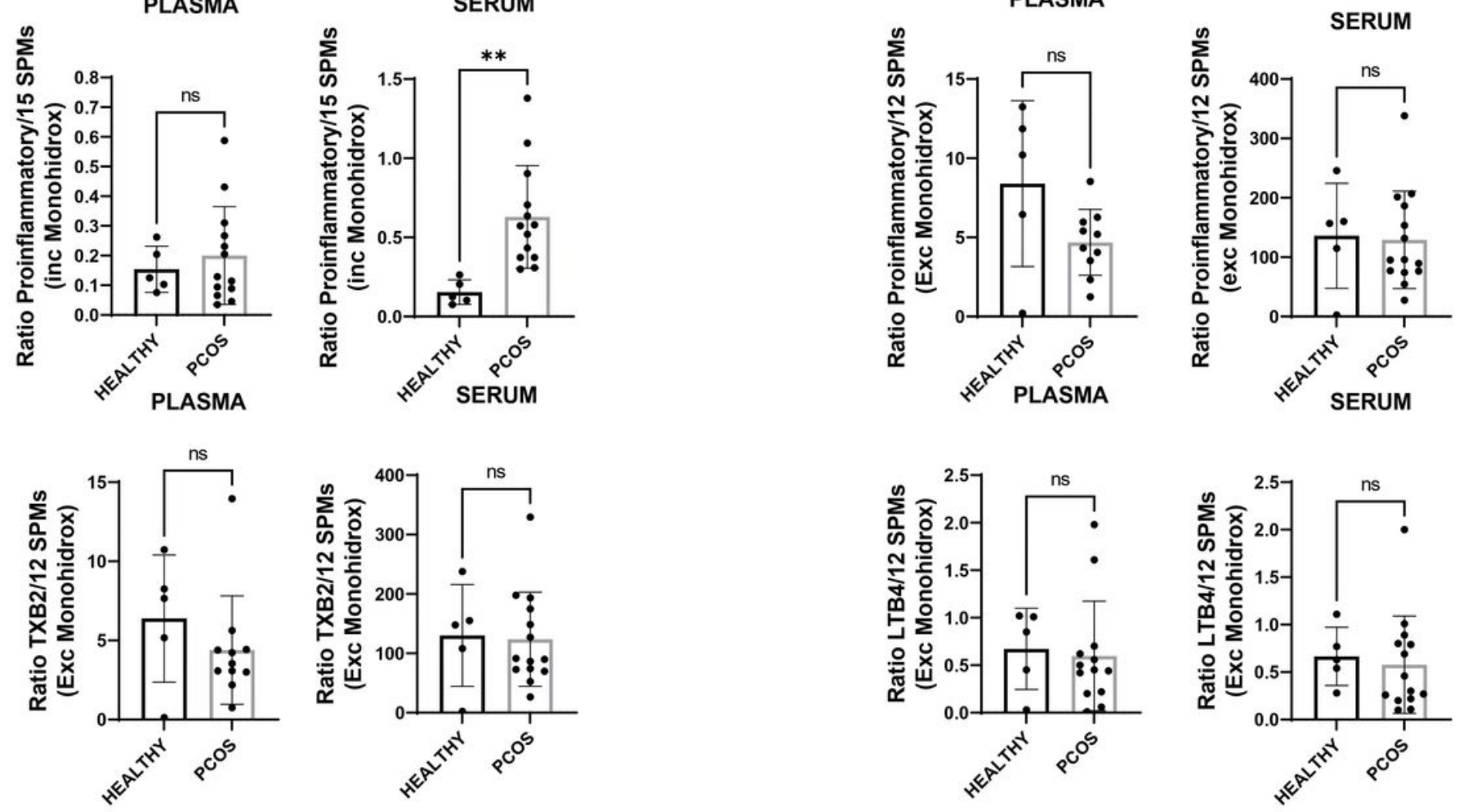

Figure 3

Results of the ratio pro-inflammatory parameters / SPMs including the monohydroxylates in the serum compared to the plasma. 
Figure 4
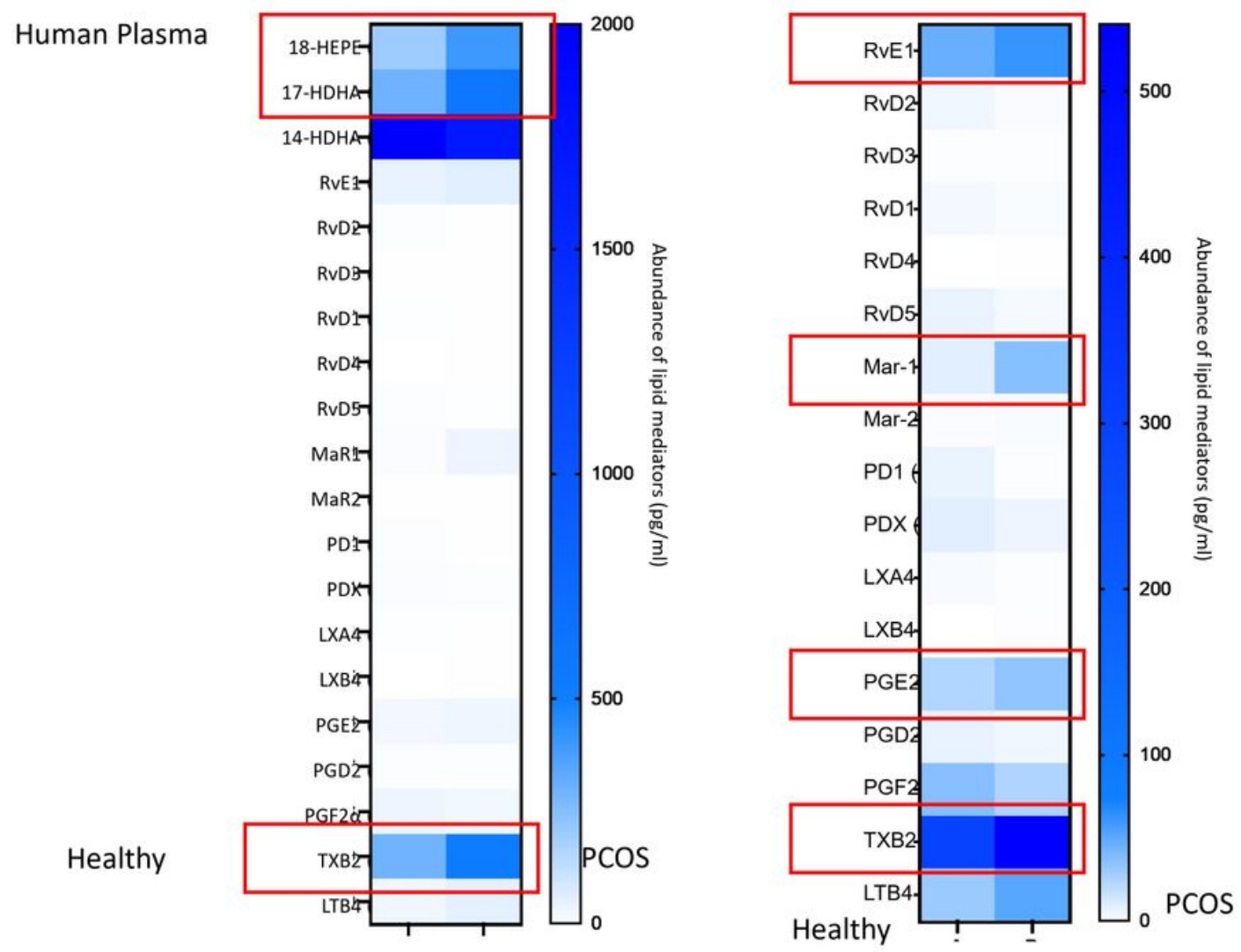

Healthy $n=5$

PCOS $n=15$

\section{Figure 4}

Heat map results in $\mathrm{pg} / \mathrm{ml}$ of the human plasma analyses of the healthy and PCOs patients.

Figure 5 Human Serum

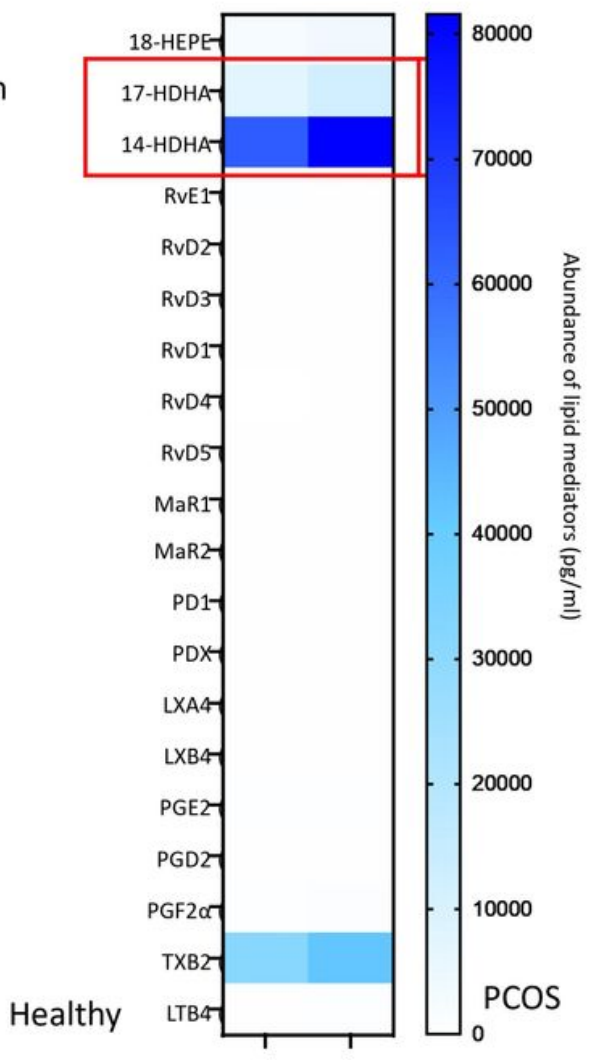

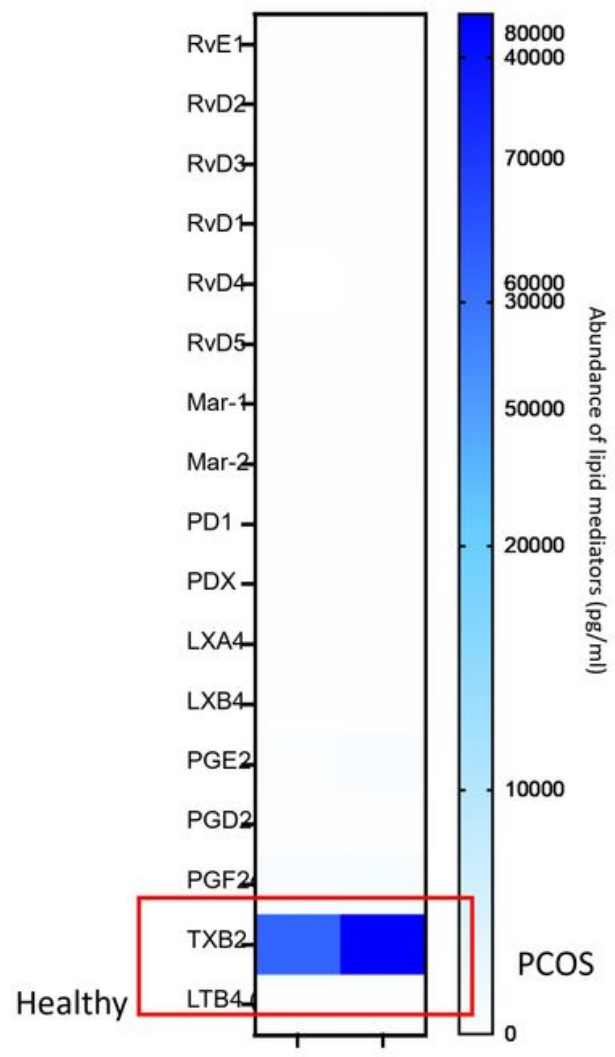

Healthy $n=5$

PCOS $n=15$ 
Figure 5

Heat map results in $\mathrm{pg} / \mathrm{ml}$ of the human serum analyses of the healthy and PCOs patients. 